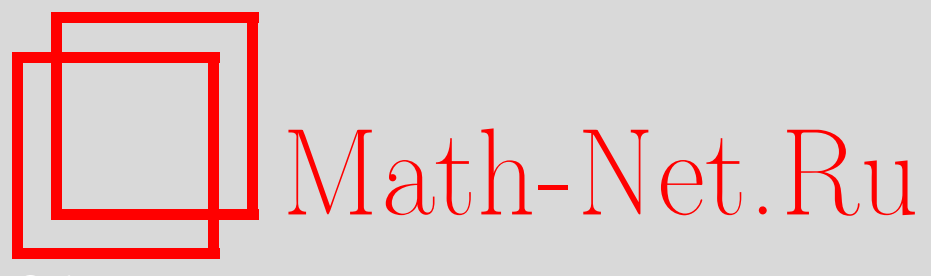

А. А. Давыдов, Т. С. Шуткина, Оптимизация циклического процесса с дисконтированием по его средней временной выгоде, УМН, 2009, том 64, выпуск 1, 143-144

DOI: https://doi.org/10.4213/rm9277

Использование Общероссийского математического портала Math-Net.Ru подразумевает, что вы прочитали и согласны с пользовательским соглашением http: //www. mathnet.ru/rus/agreement

Параметры загрузки:

IP: 3.85 .7 .115

26 апреля 2023 г., $12: 20: 10$

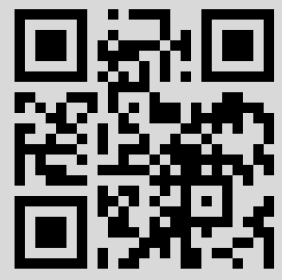




\title{
Оптимизация циклического процесса с дисконтированием по его средней временной выгоде
}

\author{
А. А. Давыдов, Т. С. Шуткина
}

Циклический процесс моделируется управляемой системой на окружности, задаваемой полем скоростей $v$, гладко зависящим от точки $x$ окружности и управляющего параметра. Предполагается, что этот параметр пробегает гладкое компактное многообразие (или объединение таковых) и принимает не менее двух различных значений, а все допустимые скорости положительные, т. е. $v>0$. Допустимым движением системы называется абсолютно непрерывное отображение $x: t \mapsto x(t)$ отрезка временной оси в фазовое пространство, в точках дифференцируемости которого его производная лежит в выпуклой оболочке множества допустимых скоростей этой точки. Цикл с периодом $T, T>0$, - это допустимое движение $x$ такое, что $x(t+T) \equiv x(t)$. При наличии непрерывной плотности выгоды $f$ на окружности выбор циклического процесса с максимальной средней временной выгодой за один оборот $\frac{1}{T} \int_{0}^{T} f(x(t)) d t \rightarrow \max$ является одной из важных задач оптимального управления. В. И. Арнольд показал, что такой цикл существует, а соответствующее ему движение устроено просто - оно использует максимальные и минимальные допустимые скорости на участках, где плотность выгоды меньше или больше максимальной средней временной выгоды за цикл [1]-[3]. В этой работе аналогичный результат получен для циклов при наличии положительного дискаунта $\sigma$, т. е. когда в функционале под интегралом плотность выгоды умножается на $e^{-\sigma t}$. Следуя В. И. Арнольду [1], мы используем положительность допустимых скоростей и переписываем задачу в виде

$$
\int_{0}^{2 \pi} \exp \left\{-\sigma \int_{0}^{x} \rho(z) d z\right\} f(x) \rho(x) d x\left(\int_{0}^{2 \pi} \rho(x) d x\right)^{-1} \rightarrow \max ,
$$

где для допустимого движения $x=x(t)$ плотность $\rho$ задается как $\rho(x(t))=1 / \dot{x}(t)$ всюду, где производная $\dot{x}(t)$ определена, т. е. почти всюду на окружности, а в остальных точках эта плотность может быть задана произвольно. Здесь 0 и $2 \pi$ - это начальная и конечная точки цикла соответственно. В такой формулировке задачи нам необходимо найти измеримую плотность $\rho$, доставляющую максимум функционала (1) и удовлетворяющую ограничениям

$$
r_{1} \leqslant \rho \leqslant r_{2},
$$

где $r_{1}$ и $r_{2}$ - положительные функции, равные обратным значениям максимума и минимума допустимой скорости соответственно. Измеримую плотность $\rho$, удовлетворяющую ограничениям (2), будем называть допустимой. Рассмотрим (1) как линейный функционал $A_{\rho}$ на пространстве непрерывных плотностей выгоды.

ПредлОЖениЕ 1. Для непрерывнъх плотности $f$ и положителъных функций $r_{1}, r_{2}$ и любой допустимой плотности $\rho$ справедлива оченка $\left|A_{\rho}(f)\right| \leqslant m_{2} M / m_{1}$, где $m_{1}=\min \left\{r_{1}(x), x \in[0,2 \pi]\right\}, m_{2}=\max \left\{r_{2}(x), x \in[0,2 \pi]\right\}, M=\max \{|f(x)|, x \in[0,2 \pi]\}$.

Теорема 2 (теорема существования). Для непрерывной плотности выгоды $f$ $u$ непрерывных положительных функиий $r_{1}, r_{2}$ существует допустимая плотность $\rho_{\max }$, доставляющая точную верхнюю грань $A$ значений функционала (1) по всем допустимым плотностям.

ДокАЗАтЕльство. Пусть для последовательности $\rho_{n}$ допустимых плотностей $A_{\rho_{n}}(f) \rightarrow A$ при $n \rightarrow \infty$. Для функций $\varphi_{n}(x)=\int_{0}^{x} \rho_{n}(z) d z, x \in[0,2 \pi]$, имеем

$$
m_{1}(y-x) \leqslant \varphi_{n}(y)-\varphi_{n}(x) \leqslant m_{2}(y-x)
$$

Работа выполнена при поддержке РФФИ (гранты № 06-01-00661-а и НШ-700.2008.1). 
для любых $x, y \in[0,2 \pi], x \leqslant y$, ибо $\rho_{n}$ удовлетворяет (2). Все $\varphi_{n}$ - липшицевы функции с одной и той же константой $m_{2}$, в частности, их совокупность равностепенно непрерывна на отрезке $[0,2 \pi]$ и ограничена. В силу теоремы Арцела-Асколи существует подпоследовательность этих функций, равномерно сходящаяся на $[0,2 \pi]$ к некоторой функции $\varphi_{\infty}$. Переходя к пределу в соотношении $(3)$, получим, что $\varphi_{\infty}$ также удовлетворяет (3). В частности, $\varphi_{\infty}$ абсолютно непрерывна, ее производная существует почти всюду и удовлетворяет условию (2) в каждой из точек своего существования. Следовательно, существует искомая плотность $\rho_{\max }$, которая в силу $(1)$ совпадает почти всюду с производной функции $\varphi_{\infty}$.

ТЕорема 3 (необходимое условие экстремума). Если для непрерывной функции $f$ допустимая плотность $\rho$ доставляет максимум А функиионала (1), то в любой точке $x$, где $\rho$ является производной своего интеграла, значение

$$
S(x):=\exp \left\{-\sigma \int_{0}^{x} \rho(z) d z\right\} f(x)-\sigma \int_{x}^{2 \pi} \exp \left\{-\sigma \int_{0}^{y} \rho(z) d z\right\} f(y) \rho(y) d y-A
$$

является неположительным, неотрицательным или равным нулю, если $\rho(x)$ равно $r_{1}, r_{2}$ или принадлежит интервалу $\left(r_{1}, r_{2}\right)$ соответственно.

ДоказАтельство. Не нарушая общности, считаем, что $x \in(0,2 \pi)$ (для граничных значений $x$ рассуждения аналогичны). Возьмем достаточно малое положительное $\nu$ такое, что интервал $[x, x+\nu]$ принадлежит $(0,2 \pi)$, и рассмотрим плотность $\tilde{\rho}$ такую, что разница $\tilde{\rho}-\rho$ равна малой константе $h \neq 0$ на этом интервале и нулю вне него. Очевидно, что плотности $\rho$ и $\tilde{\rho}$ дают периоды движения $T=\int_{0}^{2 \pi} \rho(x) d x$ и $\widetilde{T}=T+h \nu$ соответственно. Вычисляя теперь разность $\Delta=A_{\tilde{\rho}}(f)-A_{\rho}(f)$, получаем $\Delta=S(x) \times h \nu / T+\cdots$, где многоточием обозначены слагаемые более высокого порядка по $h$ и $\nu$. Так как $T>0$, то при малых $h \neq 0$ и $\nu>0$ знак этой разности определяется знаками $h$ и $S$, если $S \neq 0$. Для оптимальной плотности $\rho$ в каждой точке $x$, где $\rho$ является производной своего интеграла, разность $\Delta(x)$ должна быть неотрицательной. Следовательно, значение $S(x)$ должно быть неположительно, неотрицательно или равно нулю, если значение $\rho(x)$ равно $r_{1}(x), r_{2}(x)$ или принадлежит интервалу $\left(r_{1}(x), r_{2}(x)\right)$ соответственно, так как $h$ может принимать только неположительные, только неотрицательные и как те, так и другие значения соответственно.

При дифференцируемой плотности $f$ функция $S$ также дифференцируема и нули производных этих двух функций одинаковы. Отсюда и из теоремы 3 вытекает

Теорема 4. Для дифференцируемой плотности выгоды $f$ с конечным числом $k$ критических точек и для непрерывных положительных функи,ий $r_{1}, r_{2}$ допустимую плотность $\rho$, доставляюшую максимум в (1), можно взять кусочно непрерывной функиией, принимающей значение $r_{1}$ либо $r_{2}$ внутри любого интервала, не содержащего нули функиии переключения $S$ и концевые точки $0,2 \pi$ ицкла.

Авторы благодарны В. И. Данченко за обсуждения результатов этой работы.

\section{Список литературы}

[1] В. И. Арнольд, Функи. анализ и его прил., 36:2 (2002), 1-11. [2] А. А. Давыдов, Тр. МИАН, 250, Наука, М., 2005, 79-94. [3] А. А. Davydov, H. Mena-Matos, Singularities in geometry and topology, World Sci. Publ., Hackensack, NJ, 2007, 598-628.

\section{А. А. Давыдов (А. А. Davydov)}

Владимирский государственный университет

E-mail: davydov@vlsu.ru

\section{T. С. Шуткина (T. S. Shutkina)}

Владимирский государственный университет

E-mail: shutkina@vlsu.ru
Представлено В. М. Закалюкиным Принято редколлегией 15.01.2009 